\title{
Avaliação do Prontuário Médico de um Hospital Universitário
}

\section{Evaluation of a University Hospital Medical Record}

\author{
Célia Regina de Oliveira Garritano ${ }^{\mathrm{I}}$ (iD \\ Felipe Holanda Junqueira ${ }^{\mathrm{I}}$ (D) \\ Ely Felyppy Soares Lorosa ${ }^{\mathrm{I}}$ (D) \\ Mayara Sanae Fujimoto ${ }^{\mathrm{I}}$ (D) \\ Wallace Hostalacio Avelar Martins ${ }^{\mathrm{I}}(\mathrm{D})$
}

\section{Palavras chave}

- Prontuário médico.

- Avaliação.

- Educação médica.

Introdução: Este trabalho analisa a qualidade dos prontuários médicos dos pacientes internados no Hospital Universitário Gafrée e Guinle. Métodos: Foram selecionados aleatoriamente 100 prontuários da clínica médica e da clínica cirúrgica de pacientes internados nesse Hospital. A análise dos prontuários consistiu na ausência ou presença, dos seguintes tópicos: ficha de identificação do paciente, laudo médico, boletim de internação e alta hospitalar, resumo de internação, evolução médica, evolução da enfermagem, prescrição médica, boletim operatório, boletim da anestesia, laudo do exame histopatológico. Quando havia estes tópicos, verificou-se ainda se eles estavam adequados ou inadequados. Nos prontuários da clínica médica excluíram-se o boletim operatório e da anestesia, laudo do exame histopatológico e os demais tópicos relacionados à cirurgia. Em cada prontuário foi observado se todos os itens citados estavam presentes, preenchidos corretamente, escritos de forma legível, e se havia abreviaturas, siglas e sinais impróprios. No caso de esses itens estarem presentes nos prontuários avaliou-se a adequação ou inadequação deles. Os resultados das ambas as clínicas foram comparados para a análise de qual serviço apresentou um melhor desempenho. Para avaliar o desempenho do hospital todos os itens foram analisados em relação à presença, ausência, adequação ou inadequação e não aplicabilidade. A análise dos dados foi realizada por meio de tabelas e gráficos e a análise estatística pelo teste não paramétrico MannWhitney. Resultados: Durante a pesquisa nem todos os itens foram encontrados e, alguns casos, estavam preenchidos inadequadamente. Além disso, constatou-se a falta de ordenação deles. Do total da amostra analisada, 85,5\% estavam legíveis e $32 \%$ foram considerados inadequados, sendo 37 prontuários da clínica cirúrgica e 27 da clínica médica. Os resultados dos exames complementares foram encontrados em apenas 55,5\% da amostra. Conclusão: A elaboração dos prontuários no Hospital Universitário Gaffrée e Guinle é deficiente com vários itens incompletos e/ou ausentes. 


\section{KEY-WORDS}

- Medical Record.

- Evaluation.

- Medical Education.

Recebido em $25 / 9 / 19$

Aceito em 8/10/19

\section{INTRODUÇÃO}

A Resolução no 1.638/2002 do Conselho Federal de Medicina (CFM) define o prontuário médico como um documento único estabelecido por um agregado de informações, sinais e imagens registrados, elaborado a partir de fatos, eventos e quadros sobre a saúde do paciente e a assistência a ele prestada, de âmbito legal, sigiloso e científico ${ }^{1,2}$. Além disso, o Código de Ética Médica de 1988 no capítulo V, artigo 69, decreta que é vedado ao médico "deixar de elaborar prontuário médico para cada paciente, reiterando o valor de informar e dar transparência ao instrumento"3.

O primeiro relatório conhecido foi feito por um médico egípcio entre 3000 e 2500 a.C. ${ }^{4}$, e desde então vem sendo realizado com o objetivo de garantir a comunicação entre os componentes da equipe de saúde que direcionará a conduta, como documento que demonstra a evolução do paciente e também com caráter de assistência, ensino, pesquisa, jurídico e financeiro, para a avaliação de gastos hospitalares ${ }^{5}$.

Em geral ele possui falhas em sua formulação apresentando-se muitas vezes incompleto ou ilegível. Em um hospital universitário, esse documento deveria obrigatoriamente ser exemplar para a capacitação dos profissionais da área da saúde, que deveriam perpetuar essa prática pouco valorizada, vista por muitos apenas como empecilhos burocráticos. Uma forma de amenizar esse problema e diminuir o tempo de execução dos profissionais pode ser facilitada pela informatização da área médica por meio do Prontuário Eletrônico do Paciente (PEP) ${ }^{5}$. No entanto, o grau de desenvolvimento tecnológico dos serviços de saúde pública do país, por causa dos problemas econômico-financeiros e culturais, como a não capacitação de parte dos possíveis usuários e a falta de acesso ao software mais adequado, é muito heterogêneo. Na maioria dos serviços públicos, predominam os modelos em formato tradicional ou suporte em papel, mas que ainda assim não perdem seu grau de importância. Atualmente observa-se uma grande crise nos hospitais universitários. Como fazem

\section{ABSTRACT}

Introduction: To analyze the quality of the medical records of patients admitted to the Gafrée e Guinle University consisted in the absence or presence and, if present, whether the following items are adequate or inadequate: patient identification file; medical report; hospitalization and discharge reports; hospital admission summary; medical evolution report; nursing evolution report; medical prescriptions; operative report; anesthesia report; histopathological test report. In the Medical Clinic records the operative and anesthesia reports, histopathological test report and other topics related to surgery were excluded. In each medical report it was verified whether the aforementioned items were present, correctly filled out, using legible handwriting, and whether there wer inadequate abbreviations, symbols and signs. The results were compared in both clinics to analyze which service serformance of the hospital, all items were analyzed for their using tables and graphs, and the statistical analysis was performed by the non-parametric Mann-Whitney Test. Results: Not all items were found nor filled out, in addition to their lack of order. Of the total analyzed sample $85.5 \%$ were legible and $32 \%$ were considered inadequate, being 37 medical records from the Surgical Clinic and 27 from the Medical Clinic. The results of the additional tests were found in only 55.5\% of the sample Conclusion: The formulation of Gaffrée and Guinle University Hospital medical records is inadequate, showing multiple incomplete and/or absent items. 
Evolução da Enfermagem: assinatura e carimbo.

Prescrição médica: nome do paciente, número de matrícula, enfermaria, data, assinatura e carimbo do médico.

Boletim operatório: nome do paciente, número de matrícula, enfermaria, data, horário, descrição da intervenção, identificação do cirurgião e auxiliares, assinatura do cirurgião e carimbo.

Boletim da anestesia: nome do paciente, número de matrícula, enfermaria, data, horário, materiais e medicamentos utilizados, evolução durante a cirurgia, assinatura do profissional e carimbo.

Histopatológico: laudo caso tenha sido realizado algum procedimento cirúrgico.

Em cada prontuário observou-se se todos os itens citados estavam presentes, preenchidos corretamente, escritos de forma legível e se havia abreviaturas, siglas e sinais impróprios.

Para a avaliação dos prontuários da clínica médica excluíramse os boletins operatório e da anestesia, e histopatológico e tópicos referentes à cirurgia.

Posteriormente os prontuários da clínica médica e da cirúrgica foram comparados para verificar qual serviço produziu um prontuário mais completo.

Vale ressaltar também que os dados dos pacientes e da equipe técnica responsável permanecerão em sigilo, uma vez que o objetivo da pesquisa é fazer uma análise dos registros e não dos sujeitos envolvidos.

A análise dos dados foi realizada por meio de tabelas e gráficos e a análise estatística pelo teste não paramétrico Mann-Whitney.

\section{RESULTADOS}

$\mathrm{Na}$ análise estatística todas as amostras apresentaram uma distribuição normal pelo método Kolmogorov Smirnov (KS). Alguns itens não foram analisados estatisticamente por conta do pequeno número de variáveis.

O formulário de laudo médico foi encontrado em 184 (92\%) do total da amostra, estando ausente em nove prontuários da clínica cirúrgica e em sete da clínica médica. Não se encontrou o boletim de internação e alta em apenas um $(0,5 \%)$ prontuário do total analisado.

O preenchimento do laudo médico foi considerado adequado em 140 (70\%) do total analisado, inadequado em 44 (22\%) e em 16 (8\%) prontuários não constava o preenchimento. Em relação do boletim de internação e alta o preenchimento foi considerado adequado em $179(89,5 \%)$ do total da amostra, inadequado em 20 (10\%) e ausente em apenas um (0,5\%). Ambas as clínicas apresentaram resultados semelhantes. (Tabela 1)

A análise estatística da comparação dos resultados desses dois itens das duas clínicas também mostrou não ser significativa pelos testes de Mann-Whitney ( $\mathrm{p}>0,8260)$ e $\mathrm{t}(\mathrm{p}>0,9999)$.

A ordenação das folhas que compõem o prontuário foi considerada como adequada em 181 (90,5\%) do total da amostra e os resultados de ambas as clínicas analisadas não mostraram diferença entre elas.

A ficha da história da internação estava presente em 182 (91\%) prontuários e não foi encontrada em 18 (9\%), sendo observada em 96 prontuários da clínica médica e em 86 da clínica cirúrgica.

A ficha de evolução médica foi observada em 184 (92\%) do total analisado, não sendo encontrada em apenas dois prontuários da clínica médica, enquanto na clínica cirúrgica isto aconteceu em 14 prontuários (Gráfico 1).

Com relação à ficha de evolução de enfermagem entre todos os prontuários analisados ela foi encontrada em 185 (92,5\%) e não houve diferença na comparação entre as duas clínicas.

A folha de prescrição médica foi encontrada em 185 (92,5\%) prontuários. Constatou-se a falta dessa informação em sete prontuários da clínica médica e em 8 da clínica cirúrgica.

Ao analisarmos os exames complementares, notamos que em apenas 111 (55,5\%) do total dos prontuários eles estavam presentes. Quando se analisaram separadamente ambos os serviços, obteve-se o seguinte resultado: 86 na clínica médica e apenas 25 na cirurgia. A análise estatística da comparação dos resultados apresentados por ambos os serviços não foi significativa pelo teste de Mann-Whitney $(\mathrm{p}=0,06667)$ nem pelo teste $\mathrm{t}(\mathrm{p}=0,8225)$.

Ao avaliarmos o item exame histopatológico em somente 106 prontuários constatamos a real indicação da presença desses resultados. Desse total não encontramos o resultado do exame em quase $90 \%$ dos prontuários.

Os boletim de cirurgia, de anestesia e de material de sala cirurgia são tópicos exclusivos dos prontuários cirúrgicos. Entre todas as enfermarias cirúrgicas o boletim de cirurgia foi identificado em 97 prontuários, o de anestesia em 95 e o de material de sala em 95. A análise estatística dos dados de preenchimento das enfermarias cirúrgicas pelo teste de ANOVA não foi significativo ( $\mathrm{p}=0,9990)$. O mesmo ocorreu com os resultados do grupo de não preenchimento dos referidos boletins com o mesmo teste, o que foi considerado não significativo $(\mathrm{p}=0,7841)$. Avaliamos

\begin{tabular}{|c|c|c|c|c|}
\hline \multicolumn{5}{|c|}{$\begin{array}{l}\text { Resultados do preenchimento dos formulários de lau } \\
\text { boletim de internação e alta }\end{array}$} \\
\hline & \multicolumn{2}{|c|}{$\begin{array}{l}\text { Preenchimento } \\
\text { laudo médico }\end{array}$} & \multicolumn{2}{|c|}{$\begin{array}{l}\text { Preenchimento } \\
\text { internação e alta }\end{array}$} \\
\hline & Cirurgia & Clínica & Cirurgia & Clínica \\
\hline Adequado & 64 & 76 & 89 & 90 \\
\hline Inadequado & 27 & 17 & 10 & 10 \\
\hline Ausente & 9 & 7 & 1 & 0 \\
\hline
\end{tabular}

Fonte: Elaborada pelos autores

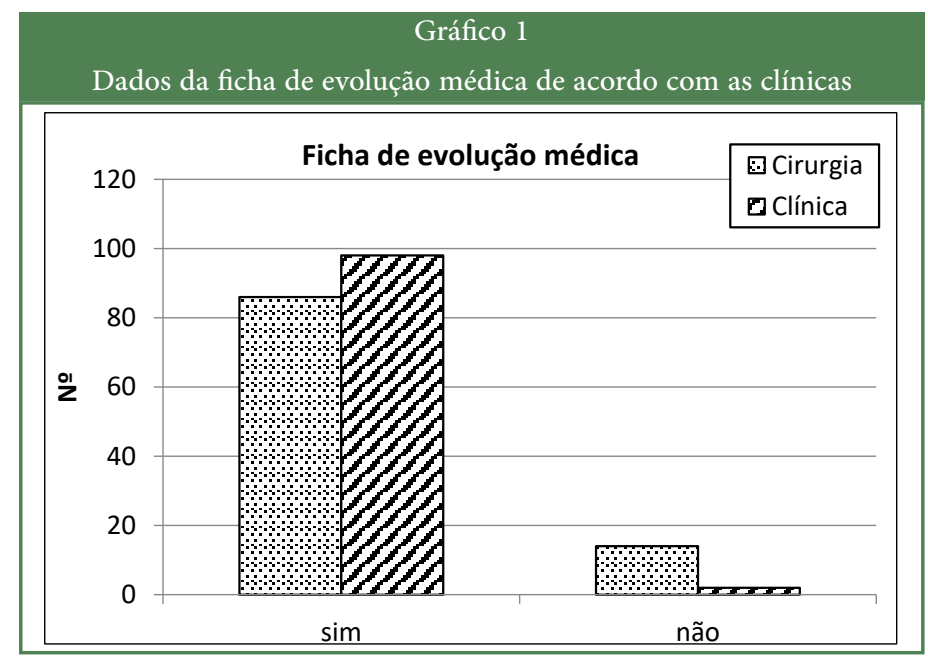

Fonte: Elaborado pelos autores 
também todos os itens comparando a variável "preenchimento" com o "não preenchimento" dos formulários específicos de todos os serviços de cirurgia. A análise de ANOVA mostrou um resultado muito significativo $(p=0,0009)$.

Do total de prontuários analisados, 171 (85,5\%) estavam legíveis, aspectos observados em 87 prontuários da clínica médica e em 84 da cirúrgica. A análise estatística desse item não foi significativa pelo teste de Mann-Whitney $(p>0,9999)$ nem pelo teste $t(p>0,9999)$.
As respostas de cada item foram agrupadas, e podemos observar na Tabela 2 que a clínica cirúrgica apresentou uma média de 75 prontuários adequados enquanto a clínica médica obteve 84 .

A análise estatística da comparação desse item não foi significativa pelo teste de Mann-Whitney ( $\mathrm{p}>0,9999)$ nem pelo teste $\mathrm{t}(\mathrm{p}>0,9999)$.

Com o objetivo de traçar o perfil do hospital juntamos os resultados de rodos os itens avaliados de ambos os serviços que são mostrados no Gráfico 2.

\section{Tabela 2}

Dados das clínicas cirúrgica e médica dos itens avaliados, exceto os que se referem exclusivamente à cirurgia.

\section{CIRURGIA}

\begin{tabular}{|c|c|c|c|c|c|c|c|c|}
\hline & Sim & Não & Inadequado & Não se aplica & Sim & Não & Inadequado & Não se aplica \\
\hline Laudo médico & 91 & 9 & 0 & 0 & 93 & 7 & 0 & 0 \\
\hline Preenchimento laudo & 64 & 9 & 27 & 0 & 76 & 7 & 17 & 0 \\
\hline $\begin{array}{l}\text { Boletim de } \\
\text { internação/alta }\end{array}$ & 99 & 1 & 0 & 0 & 100 & 0 & 0 & 0 \\
\hline Preenchimento boletim & 89 & 1 & 10 & 0 & 90 & 0 & 10 & 0 \\
\hline Ordenação das folhas & 90 & 10 & 0 & 0 & 91 & 9 & 0 & 0 \\
\hline História da internação & 86 & 14 & 0 & 0 & 96 & 4 & 0 & 0 \\
\hline Evolução médica & 86 & 14 & 0 & 0 & 98 & 2 & 0 & 0 \\
\hline Evolução da enfermagem & 92 & 8 & 0 & 0 & 93 & 7 & 0 & 0 \\
\hline Prescrição médica & 92 & 8 & 0 & 0 & 93 & 7 & 0 & 0 \\
\hline Exames complementares & 25 & 75 & 0 & 0 & 86 & 14 & 0 & 0 \\
\hline Legível & 84 & 16 & 0 & 0 & 87 & 13 & 0 & 0 \\
\hline Histopatológico & 5 & 56 & 0 & 39 & 6 & 39 & 0 & 55 \\
\hline Média & 75,3 & 18,4 & 3,1 & & 84,1 & 9,1 & 2,3 & \\
\hline Desvio padrão & 29,7 & 22,8 & 8,1 & & 25,4 & 10,4 & 5,5 & \\
\hline
\end{tabular}

Fonte: Elaborada pelos autores

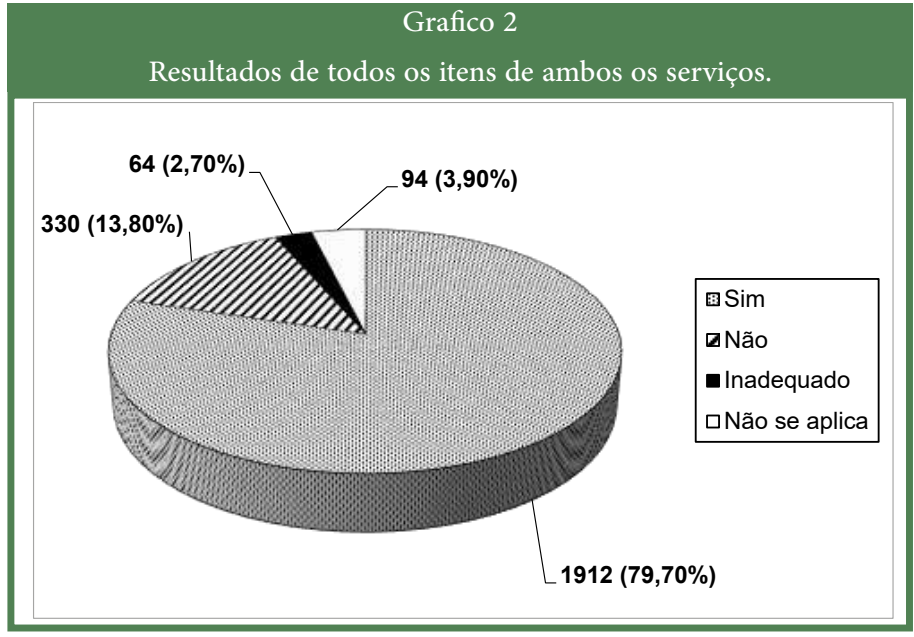

Fonte: Elaborado pelos autores

\section{DISCUSSÃO}

O prontuário médico é um documento elaborado pelo profissional de saúde no qual devem constar, de forma organizada e concisa, todos os dados relativos ao paciente. No prontuário os itens obrigatórios são: identificação do paciente, anamnese, exame físico, hipóteses diagnósticas, diagnóstico definitivo e tratamento efetuado ${ }^{6}$. Esse documento é de propriedade do paciente que tem total direito de acesso e pode solicitar uma cópia ${ }^{5}$. É importante ressaltar que quando realizado de maneira adequada ele pode contribuir para o ensino, a pesquisa, a elaboração de censos, propostas de assistência à saúde pública e a avaliação da qualidade da assistência médica prestada ${ }^{1}$.

Na acreditação hospitalar o Ministério da Saúde estabelece como padrão de nível 1 o arquivo médico cujos prontuários sejam legíveis, estejam assinados pelo médico assistente e apresentem uma sequência lógica do seu conteúdo ${ }^{1}$. 
No laudo médico devem constar todas as informações consideradas obrigatórias pelo CFM: o estabelecimento solicitante e executante, identificação do profissional solicitante assinatura e carimbo e autorização do gestor ${ }^{10}$.

Neste trabalho observamos que esse item não estava presente em $8 \%$ da amostra. Em $22 \%$ constatamos que o preenchimento estava inadequado. Os principais pontos que motivaram a inadequação apresentada foram a ilegibilidade e a presença de siglas e abreviaturas. Isso poderá prejudicar a comunicação entre membros da equipe de saúde não terão condições de traduzir o significado das inúmeras siglas e abreviaturas ali contidas. Além disso, o prontuário tem que ser inteligível ao paciente que, sendo leigo, não é obrigado a decifrar siglas de conhecimento exclusivo do médico/especialista ${ }^{6}$.

A ordenação correta permite ao médico acompanhar a evolução do paciente, bem como compreender a história cronológica de cada internação. É primordial riscar os espaços vazios para impedir as anotações fora de ordem temporal ${ }^{6}$. Em nossa amostra observou-se que em 90,5\% dos prontuários analisados a ordenação das folhas foi satisfatória, não havendo divergência significativa entre as clínicas estudadas. Como o Hospital Universitário Gaffrée e Guinle é uma instituição de ensino, com residentes, internos e graduandos de Medicina, Enfermagem, Psicologia, Fisioterapia e Nutrição que também escrevem no prontuário, o paciente é assistido por mais de um profissional que utiliza esse registro. Entretanto por muitas vezes, os profissionais deixam as folhas completamente desordenadas não se atentando para a importância dessa atitude. Em outros casos, como os pacientes podem ser transferidos de um local para outro - por exemplo, do centro de terapia intensiva (CTI) para enfermaria, os prontuários não são organizados cronologicamente.

Nos prontuários em suporte de papel, é obrigatória a legibilidade da letra do profissional que atendeu o paciente (Resolução CFM $\mathrm{n}^{\circ}$ $1.638 / 2002)^{1,8,10}$. Devemos ter atenção também ao excesso de abreviações, siglas e sinais impróprios, que podem causar uma falsa compreensão, ou siglas restritas às especialidades e aquelas com várias interpretações ${ }^{3}$. Quanto à legibilidade constatou-se um resultado satisfatório em 85,5\% dos prontuários. Ainda que percentualmente esse resultado não cause um impacto grande à primeira vista, devemos entender que o prontuário médico representa o atendimento de cada paciente. Salientamos esse documento também serve como matéria-prima para pesquisas científicas bem como para a análise da própria instituição sobre o serviço prestado.

Anexar a segunda via do pedido de exames ao prontuário é importante para o diagnóstico, o tratamento e a evolução do paciente. Podemos pontuar também que alguns desses exames são imprescindíveis para a realização de determinados procedimentos cirúrgicos e a sua ausência pode impossibilitar uma intervenção cirúrgica no momento mais apropriado. Deve haver uma margem lateral para que seja possível anexar as folhas dos laudos dos exames e inserir a assinatura e o carimbo do médico emitente com número de inscrição no conselho regional de classe ${ }^{6}$. De acordo com a Resolução do CFM n ${ }^{\circ}$ 1.639/2002, o tempo mínimo para o arquivamento de laudos é de 20 anos $^{8}$. Legalmente é um instrumento de defesa jurídica em casos de perda, extravios das solicitações e não realização do exame ${ }^{(6)}$. $\mathrm{Na}$ pesquisa as informações sobre os exames foram encontradas em um pouco mais da metade dos prontuários analisados, havendo uma grande diferença entre a clínica médica e a cirurgia, $86 \%$ e $25 \%$ respectivamente. Notamos também que uma grande parte dos pacientes já chega portando exames realizados em outros locais, incluindo a rede privada de saúde, e reclamam a sua posse após o tratamento. Outro problema constatado foi a demora na obtenção dos resultados dos exames realizados. Em geral, é necessário aproximadamente um mês para obtenção dos laudos referentes às tomografias computadorizadas (TC) do serviço de radiologia. Essa demora dificulta que sejam anexados aos prontuários, sobretudo quando os pacientes já receberam alta hospitalar.

Observou-se o mesmo comportamento na liberação dos laudos dos exames histopatológicos - sem dúvida trata-se do dado mais alarmante obtido nesta pesquisa. Do total da amostra, esses laudos estavam anexados em apenas 11 prontuários. Mais uma vez o motivo é a demora a obtenção dos laudos, além do descuido do profissional em não anexálos posteriormente. Os pedidos entregues no laboratório do Hospital Universitário Gaffrée e Guinle devem conter o termo "urgente" para que os resultados dos procedimentos possam ficar prontos em menos de 30 dias. Segundo o Parecer da Sociedade Brasileira de Patologia $n^{\circ}$ 06/2000, por equivalência, o histopatológico e citopatológico são exames complementares, sendo parte integrante e obrigatória em um prontuário médico, e devem receber o mesmo tratamento para arquivamento, de acordo com a legislação arquivista vigente pela Lei $n^{\circ} 8.159$, de 8 de janeiro de $1991^{11}$.

Os boletins de cirurgia, de anestesia e de material de sala cirurgia são tópicos exclusivos dos prontuários cirúrgicos. Eles são muito importantes para a administração hospitalar e gestão institucional, pois, por meio desses boletins pode-se obter o repasse das verbas do governo federal'. Esses dados também podem ser utilizados como fonte para a realização de estudos epidemiológicos e avaliação dos serviços de cirurgia. Em todas as enfermarias esses itens foram encontrados na quase totalidade dos prontuários.

A ausência da ficha de prescrição médica compromete o perfeito entendimento da evolução do quadro e da análise da qualidade do atendimento prestado ao paciente ${ }^{6}$,já que ela contém todas as informações sobre a dieta, e as medicações, além das orientações médicas destinadas à outros profissionais. Apesar de frequentes na quase totalidade dos prontuários analisados, muitas fichas não apresentavam o correto preenchimento da data e da administração da medicação, o que pode causar confusão entre os profissionais, além do risco de aplicação incorreta de medicamentos.

A ficha de evolução médica deve conter todas as informações sobre o período de internação do paciente, como a data e hora de sua realização, o exame físico, os sinais vitais no momento e aqueles registrados pela enfermagem previamente, as queixas relativas às últimas 24 horas e a conduta diária. Essa ficha Foi encontrada em $92 \%$ dos prontuários, cabendo mais uma vez à Clínica médica apresentar um melhor resultado em comparação à cirúrgica. Desse modo, a realização ineficiente dessa parte do prontuário leva a um mau entendimento da resposta do paciente às medicações, às alterações da terapia proposta e à realização de exames.

A ficha de evolução de enfermagem estava presente em quase todos os prontuários, mas ressaltamos a dificuldade no entendimento das informações por conta da ilegibilidade.

O boletim de alta hospitalar deve conter informações fundamentais do período de internação do paciente. O preenchimento desse boletim é importante para uma análise posterior seja de ordem jurídica, médica, pesquisa ou da gestão institucional. Na pesquisa nem todos os prontuários continham esse item, e em muitos casos não havia a assinatura do médico, a numeração da CID, o resumo de alta e, principalmente, o diagnóstico.

REVISTA BRASILEIRA DE EDUCAÇÃO MÉDICA

5 44 (1): e009; 2020 
"Quem tem a informação tem o poder", é uma frase atribuída a Francis Bacon. No contexto do trabalho, "poder" é investigar o diagnóstico, fazer o tratamento, gerenciar os gastos hospitalares, desenvolver trabalhos científicos, avaliar o desempenho do hospital entre outros aspectos. A carência da informação compromete todos esses itens especialmente em se tratando de um hospital universitário que depende de verbas governamentais para exercer as funções assistencial e de ensino. Os resultados deste trabalho mostram uma evidente falta de informações de diversos itens, o que pode ter comprometido a qualidade do atendimento, principalmente com a demora no diagnóstico e tratamento além de dificultar a elaboração de trabalhos científicos baseados nessas informações.

Outro fato é não constar na grade curricular da graduação na área da saúde uma disciplina que aborde a necessidade da elaboração de um prontuário adequado. Na sua maioria, os médicos, residentes e internos da área da saúde não recebem informações sobre a importância dos prontuários e nem são incentivados a produzir um prontuário que seja ideal, que é este visto apenas como uma parte burocrática do hospital e não como um elemento de estudo epidemiológico, de gestão hospitalar e de melhoria na qualidade do atendimento aos pacientes.

Assim sendo, faz-se necessária e urgente a implantação de uma metodologia que seja capaz de aprimorar o prontuário. Neste contexto a criação do prontuário eletrônico é certamente a melhor opção.

\section{CONCLUSÃO}

A análise de todos os itens evidenciou uma deficiência na elaboração dos prontuários no Hospital Universitário Gaffrée e Guinle com vários itens incompletos e/ou ausentes.

\section{REFERÊNCIAS}

1. Dall'aglio JS, Martins Z. Avaliação de dados de prontuários do módulo urgência e emergência do Hospital de Clínicas da Universidade Federal de Uberlândia. [acesso em 20 jun 2018\}. Disponível em: <https://docplayer.com.br/1799054->

2. Reis AOA, Dombi-Barbosa C, Bertolino Neto MM, Prates MML, Delfini OS de SP, Fonseca LF et al. Prontuários, para que servem? Representação dos coordenadores de equipe dos CAPSi a respeito do valor e da utilidade dos prontuários. Rev Bras Crescimento Desenvolv Hum 2009;19(3): 383-92.

3. Conselho Federal de Medicina. Código de Ética Médica (versão de 1988). [acesso em 15 jul. 2017]. Disponível em <https://portal.cfm.org.br/>
4. Carvalho LF. Serviço de arquivo médico e estatística de um hospital,

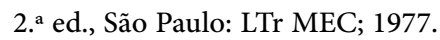

5. Fábia GS, TAVARES-NETO J. Avaliação dos Prontuários Médicos de Hospitais de Ensino do Brasil. Rev Bras Educ Med 2007;31(2): 113-26.

6. Conselho Regional de Medicina do Distrito Federal. Prontuário médico do paciente: guia para uso prático. Brasília: Conselho Regional de Medicina, 2006.

7. Código de Ética Médica. [acesso em 15 jul. 2017]. Disponível em: <http://www.portalmedico.org.br/resolucoes/cfm/1988/1 >.

8. Conselho Federal de Medicina. Resolução CFM nº 1997/2012. Diário Oficial da União de 16 de ago 2012, Seção I, 149.

9. Morais RD, Souza SAJ, Scudelari SAR, Siqueira AS, Bezerra FA; Gervásio DMS. Avaliação dos Prontuários dos Pacientes de uma Instituição Pública do Município de Paraibuna de São Paulo. Vita Sanitas, 2012 jan-dez;(6):2-16.

10. Sampaio AC. Qualidade dos prontuários médicos como reflexo das relações médico-usuário em cinco hospitais do Recife/PE. Recife. Tese [Doutorado em saúde Pública\}Fundação Oswaldo Cruz, 2010.

11. Sociedade Brasileira de Patologia. Consulta: tempo exigido para arquivar os blocos de parafina, os laudos e as lâminas dos exames histopatológicos, citológicos e requisições dos Exames. [acesso em 20 jun 2018]. Disponivel em: http://siteantigo.sbp.org.br/publicacoes/ pareceresImpressao.aspx?id=43.

\section{CONTRIBUIÇÕES DOS AUTORES}

Todos os autores participaram ativamente na elaboração do trabalho.

\section{CONFLITO DE INTERESSE}

Não há conflito de interesses.

\section{ENDEREÇO PARA CORRESPONDENCIA:}

Prof ${ }^{a}$ Célia Garritano

Rua Uruguai, 124/402. Tijuca. Rio de Janeiro, RJ.

CEP - 20510-060.

e-mail: cgarritano@gmail.com 